\title{
ON THE DYNAMICS OF THE UNIFIED CHAOTIC SYSTEM BETWEEN THE LORENZ AND CHEN SYSTEMS.
}

\author{
JAUME LLIBRE \\ Departament de Matemàtiques, Universitat Autònoma de Barcelona, \\ 08193 Bellaterra, Barcelona, Catalonia, Spain \\ jllibre@mat.uab.cat. \\ ANA RODRIGUES \\ University of Exeter, College of Engineering, \\ Mathematics and Physics, Exeter EX4 $4 Q E, U K$ \\ a.rodrigues@exeter.ac.uk
}

March 10, 2015

A one-parameter family of differential systems that bridges the gap between the Lorenz and the Chen systems was proposed by Lu, Chen, Cheng and Celikovsy. The goal of this paper is to analyze what we can say using analytic tools about the dynamics of this one-parameter family of differential systems. We shall describe its global dynamics at infinity, and for two special values of the parameter $a$ we also can describe the global dynamics in the whole $\mathbb{R}^{3}$ using the invariant algebraic surfaces of the family. Additionally we characterize the Hopf bifurcations of this family.

Keywords: Hopf bifurcation; Lorenz system; Chen system; unified chaotic system; invariant algebraic surface; Poincaré compactification.

\section{Introduction and statement of the main results}

In 1963 Lorenz (see [Lorenz, 1963]) introduced the following non-linear system in $\mathbb{R}^{3}$ of differential equations

$$
\begin{aligned}
& \dot{x}=\sigma(y-x), \\
& \dot{y}=\rho x-y-x z, \\
& \dot{z}=-\beta z+x y,
\end{aligned}
$$

for which it was observed sensitive dependence of initial conditions. For an open neighborhood of certain parameter values $(\sigma, \rho$ and $\beta)$, numerical simulations suggested the existence of a strange attractor, known now as the Lorenz attractor. The proof of the existence of a robust strange attractor for these equations was later given by Tucker [Tucker, 2002].

The following differential system in $\mathbb{R}^{3}$

$$
\begin{aligned}
& \dot{x}=a(y-x), \\
& \dot{y}=(c-a) x-x z+c y, \\
& \dot{z}=x y-b z,
\end{aligned}
$$

where $a, b, c \in \mathbb{R}$ are parameters is known as the Chen system [Chen \& Ueta, 1999]. For suitable choices of the parameters it exhibits chaotic phenomena which resembles some familiar features 
from the Lorenz system. Through these recent years the dynamics of the Chen system has been analyzed from different points of view.

Recently in [Algaba et al., 2013, Chen, 2013] it is observed that the Chen system becomes the Lorenz system doing a rescaling of the three spatial variables and of the time, namely $(x, y, z, t) \rightarrow$ $(-c x,-c y,-c z,-c t)$, which reverses the time variable if $c>0$. Then the strange attractor of the Chen system becomes a strange atractor in the Lorenz system but in backwards time, different to the classical Lorenz attractor. Moreover, both attractors take place for different values of the parameters. This observation shows that the Lorenz and the Chen systems are strongly related, and as it was mentioned in [Leonov \& Kuznetsov, 2015] both systems stimulate the developments of new methods for the analysis of the chaotic systems.

In $2002 \mathrm{Lu}$, Chen, Cheng and Celikovsy [Lu et al., 2002], introduced a one-parameter unified chaotic system that contains the Lorenz and the Chen systems as two dual systems at the two extremes of its parameter spectrum. The system introduced is

$$
\begin{aligned}
& \dot{x}=P(x, y, z)=(25 a+10)(y-x), \\
& \dot{y}=Q(x, y, z)=(28-35 a) x-x z+(29 a-1) y, \\
& \dot{z}=R(x, y, z)=x y-\frac{1}{3}(a+8) z .
\end{aligned}
$$

The differential system (1) here studied provides a continued transition from the Lorenz system (when $a=0$ ) to the Chen system (when $a=1$ ) and it is chaotic along all the values of the transition. Since the Lorenz and the Chen systems played and are playing an important role in the study of the chaotic systems in $\mathbb{R}^{3}$, it is interesting to study the dynamics of the differential system (1) which connects the mentioned two differential systems.

Later on system (1) is considered in [Lu et al., 2006] with the name unified chaotic system. So in the rest of this paper the differential system (1) will be denoted as the unified chaotic system and we shall study it for all values of $a \in \mathbb{R}$.

For a study of the differences and similarities in the analysis of the Lorenz, Chen and Lu systems see for instance [Leonov \& Kuznetsov, 2015].

In [Celikovsky et al., 1994, 2002 and 2005] the authors introduced the generalized Lorenz canon- ical form (GLCF), which contains the classical Lorenz system and the Chen system as two extreme cases, along with infinitely many chaotic systems in between. It was also shown how several other chaotic systems studied are special cases of the GLCF.

In general to describe the global dynamics of a nonlinear differential system in $\mathbb{R}^{3}$ is a hard problem, usually unsolved. The goal of this paper is to analyze what we can say using analytic tools about the dynamics of the unified chaotic system. We shall describe its global dynamics at infinity, and for two special values of the parameter $a$ we also can describe the global dynamics in the whole $\mathbb{R}^{3}$ using the invariant algebraic surfaces of the unified chaotic system. Additionally we characterize its Hopf bifurcations.

For some potential practical application of the unified chaotic system see for instance [Lin et al., 2015].

The following result shows that the dynamics in a neighborhood of the infinity for the unified chaotic system is the same as that in the Lorenz and in the Chen system, see for more details [Llibre et al., 2010] and [Llibre et al., 2012], respectively. The study of the infinity for a polynomial differential system is made using the Poincaré compactification, see section 2 for a brief introduction to such compactification.

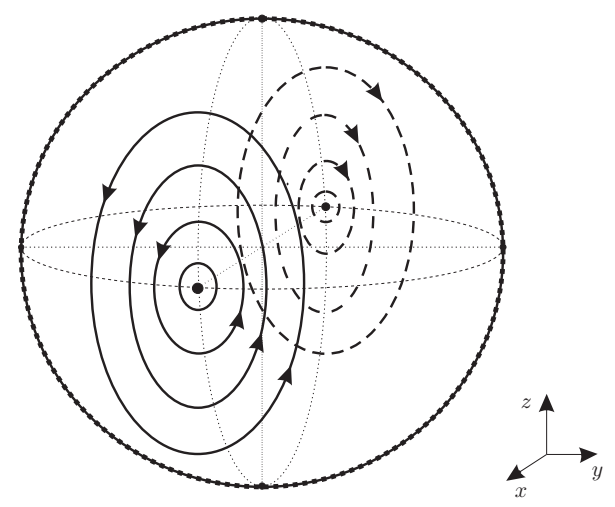

Fig. 1. Phase portrait at infinity of the unified chaotic system in the Poincaré ball.

Proposition 1.1. For all values of the parameters $a, b, c$ the phase portrait of the unified chaotic system on the sphere at infinity has two centers at the 
endpoints of the $x$-axis, the period annulus of these centers end at the circle defined by the infinity of the plane $\{x=0\}$, which is filled of equilibria, see Figure 1.

Proposition 1.1 is proved in section 2.

Let $\mathbb{R}[x, y, z]$ be the ring of the real polynomials in the variables $x, y$ and $z$. We say that $F=F(x, y, z)$ is a Darboux polynomial of system (1) if it satisfies

$$
(\nabla F) \cdot(P, Q, R)=k F,
$$

where $k=k(x, y, z)$ is a real polynomial of degree at most 1 , called the cofactor of $F(x, y, z)$ and $\nabla F$ is the gradient of $F$. If the cofactor is zero, then $F(x, y, z)$ is a polynomial first integral of system (1). If $F(x, y, z)$ is a Darboux polynomial with nonzero cofactor, then the surface $F(x, y, z)=0$ is an invariant algebraic surface, i.e. if an orbit of system (1) has a point on this surface, then the whole orbit is contained in it.

In the following proposition we study the invariant algebraic surfaces for system (1).

Proposition 1.2. The following statements hold for the unified chaotic system.

(a) If $a=-2 / 5$ then $x$ is a first integral, and the restriction of unified chaotic system to each invariant plane $x=$ constant is a linear differential system.

(b) If $a=-52 / 149$, then the unified chaotic system has the invariant algebraic surface $F(x, y, z)=149 x^{2}-380 z=0$.

(c) The cofactor of any invariant algebraic surface of a unified chaotic system is a constant.

(d) The only invariant algebraic surfaces of degree $\leq 6$ of the unified chaotic systems are the ones of statements $(a)$ and $(b)$.

Proposition 1.2 is proved in section 3.

The next result describes the global dynamics of the unified chaotic system in the Poincaré ball when the parameter $a$ takes the values $-2 / 5$ and $-52 / 149$, i.e. when the system has invariant algebraic surfaces.
Theorem 1.3. The following statements hold for the unified chaotic systems.

(a) For $a=-2 / 5$ all the planes $x=h \in \mathbb{R}$ are invariant. The unique finite equilibrium point of the unified chaotic system in the plane $x=$ $h$ is

$$
\left(h, \frac{847 h}{4 h^{2}+539}, \frac{462 h^{2}}{4 h^{2}+539}\right),
$$

a global stable focus or node if $|h|>215 / 6$ or $|h| \leq 215 / 6$, respectively. Clearly all the planes $x=h$ reach the infinity in the circle filled of equilibria.

(b) For $a=-52 / 149$ on the invariant algebraic surface $149 x^{2}-380 z=0$ there are three finite equilibria of the unified chaotic system

$$
(0,0,0),\left( \pm \frac{170 \sqrt{57}}{149}, \pm \frac{170 \sqrt{57}}{149}, \frac{4335}{149}\right) \text {. }
$$

On this surface the origin is a saddle and the other two equilibria are stable foci, the two unstable separatrices of the saddle go one to one focus and the other to the other focus. While the two stable separatrices of the saddle come spiraling from the infinity, see Figure 2. The invariant surface reaches the infinity in half of the circle filled of equilibria, more precisely at the infinity of the half-plane $x \geq$ 0. There is numerical evidence that the flow in the interior of the Poincaré ball outside the invariant algebraic surface tends in forward time to the invariant surface.

Theorem 1.3 is proved in section 3 .

The Hopf bifurcation of the Lorenz system has been studied, see for instance [Roschin, 1978, Buzzi et al., 2007]. Also the Hopf bifurcation of the Chen system has been analyzed, see for example [Li \& Chen, 2003]. Now our aim is to study the Hopf bifurcation of the unified chaotic system (1) that bridges the gap between the Lorenz system and the Chen system.

The equilibria of the unified chaotic system are

$$
\begin{aligned}
p_{0}= & (0,0,0), \\
p_{ \pm}= & ( \pm \sqrt{(9-2 a)(8+a)}, \pm \sqrt{(9-2 a)(8+a)}, \\
& 3(9-2 a)) .
\end{aligned}
$$




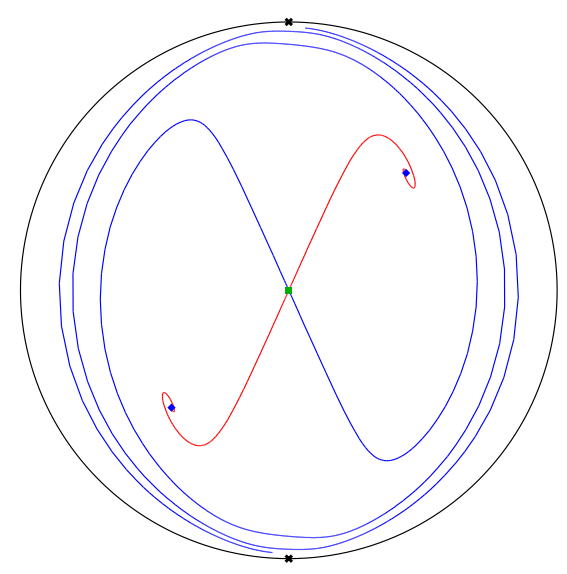

Fig. 2. Qualitative phase portrait of the unified chaotic system on the invariant algebraic surface $149 x^{2}-380 z=$ 0 .

Note that the unified chaotic system has three equilibria if $a \in(-8,9 / 2)$, two equilibria if $a=-8$ or $a=9 / 2$, and one equilibrium if $a \in \mathbb{R} \backslash[-8,9 / 2]$.

It is easy to check that the linearization of the unified chaotic system at $p_{0}$ never has a pair of conjugate purely imaginary eigenvalues, so the equilibrium point $p_{0}$ cannot exhibit a Hopf bifurcation.

The unified chaotic system is symmetric with respect to the involution $(x, y, z) \mapsto(-x,-y, z)$, i.e. it has symmetry with respect to the $z$-axis. So, if there is a Hopf bifurcation at the point $p_{-}$ there is also a Hopf bifurcation at the point $p_{+}$. Consequently our analysis will be only at the point $p_{-}$.

As we shall see later on the linearization of the unified chaotic system at $p_{-}$has a pair of conjugate purely imaginary eigenvalues and one real eigenvalue for the unique value

$$
a^{*}=-0.0136810441173477 \ldots
$$

of the parameter $a$ for which the equilibrium $p_{-}$exists. In fact $a$ is the unique real zero of a function $\varepsilon(a)$ defined later on. Therefore when $a=a^{*}$ we have the setting for a Hopf bifurcation. That is, we can expect to see a small-amplitude limit cycle bifurcating from the equilibrium point $p_{-}$. But in order that this bifurcation takes place it remains to compute the first Lyapunov coefficient $\ell_{1}\left(p_{-}\right)$of system (1) at the equilibrium $p_{-}$. When $\ell_{1}\left(p_{-}\right)<0$ the point $p_{-}$is a weak focus of the unified chaotic system restricted to the central manifold of $p_{-}$and the limit cycle that emerges from $p_{-}$is stable. In this case the Hopf bifurcation is called supercritical. When $\ell_{1}\left(p_{-}\right)>0$ the point $p_{-}$is also a weak focus of the unified chaotic system restricted to the central manifold of $p_{-}$but the limit cycle that borns from $p_{-}$is unstable. In this second case the Hopf bifurcation is called subcritical. For more details on the Hopf bifurcation see for instance the book of Kuznetsov [Kuznetsov, 2004].

The next result characterize the Hopf bifurcation in the unified chaotic system.

Theorem 1.4. The Lorenz-Chen system has a subcritical Hopf bifurcation at the equilibrium $p_{-}$ when $a=a^{*}$, and there exists a small $\varepsilon>0$ such that for $a \in\left(a^{*}-\varepsilon, a^{*}\right)$ the system has an unstable limit cycle.

Theorem 1.4 is proved in section 4 .

\section{The Poincaré compactification}

In what follows first we present a summary of the Poincaré compactification of a polynomial vector field in $\mathbb{R}^{3}$, for more details see [Cima \& Llibre, 1990].

We consider the polynomial differential system $\dot{x}=P^{1}(x, y, z), \quad \dot{y}=P^{2}(x, y, z), \quad \dot{z}=P^{3}(x, y, z)$, in $\mathbb{R}^{3}$, or equivalently its associated polynomial vector field $X=\left(P^{1}, P^{2}, P^{3}\right)$. The degree $n$ of $X$ is defined as $n=\max \left\{\operatorname{deg}\left(P^{i}\right): i=1,2,3\right\}$.

Let $\mathbb{S}^{3}=\left\{y=\left(y_{1}, y_{2}, y_{3}, y_{4}\right) \in \mathbb{R}^{4}:\|y\|=1\right\}$ be the unit sphere in $\mathbb{R}^{4}$, and $\mathbb{S}_{+}=\left\{y \in \mathbb{S}^{3}: y_{4}>\right.$ $0\}$ and $\mathbb{S}_{-}=\left\{y \in \mathbb{S}^{3}: y_{4}<0\right\}$ be the northern and southern hemispheres, respectively. We denote by $T_{y} \mathbb{S}^{3}$ the tangent space to $\mathbb{S}^{3}$ at the point $y$. We identify $\mathbb{R}^{3}$ with the tangent hyperplane $T_{(0,0,0,1)} \mathbb{S}^{3}=\left\{\left(x_{1}, x_{2}, x_{3}, 1\right) \in \mathbb{R}^{4}:\left(x_{1}, x_{2}, x_{3}\right) \in\right.$ $\left.\mathbb{R}^{3}\right\}$.

Doing central projections of the hyperplane $T_{(0,0,0,1)} \mathbb{S}^{3}$ on the sphere $\mathbb{S}^{3}$ we get two copies of our vector field $X$ on $\mathbb{S}^{3}$, one in the open northern hemisphere $\mathbb{S}_{+}$and the other in the open southern hemisphere $\mathbb{S}_{-}$. Now the equator $\mathbb{S}^{2}=\mathbb{S}^{3} \cap\left\{y_{4}=0\right\}$ plays the role of the infinity of $\mathbb{R}^{3}$. There is a unique extension of the two copies of the polynomial vector field $X$ on $\mathbb{S}_{+} \cup \mathbb{S}_{-}$to an analytic vector field $p(X)$ 
on $\mathbb{S}^{3}$. This vector field $p(X)$ on $\mathbb{S}^{3}$ is called the Poincaré compactification of $X$.

Note that the projection of $\mathbb{S}_{+} \cup \mathbb{S}^{2}$ on the hyperplane $y_{4}=0$ through $\left(y_{1}, y_{2}, y_{3}, y_{4}\right) \rightarrow$ $\left(y_{1}, y_{2}, y_{3}\right)$ is the unit closed ball centered at the origin of $\mathbb{R}^{3}=\left\{\left(y_{1}, y_{2}, y_{3}\right)\right\}$. The interior of this ball is diffeomorphic to $\mathbb{R}^{3}$ and its boundary $\mathbb{S}^{2}$ corresponds to the infinity of $\mathbb{R}^{3}$. This ball is called the Poincaré ball. $\mathbb{S}^{3}:$

We consider the following eight local charts on

$$
\begin{aligned}
& U_{i}=\left\{\left(y_{1}, y_{2}, y_{3}, y_{4}\right): y_{i}>0\right\}, \quad \text { and } \\
& V_{i}=\left\{\left(y_{1}, y_{2}, y_{3}, y_{4}\right): y_{i}<0\right\},
\end{aligned}
$$

for $i=1,2,3,4$. Then the analytical field $p(X)$ in the local chart $U_{1}$ becomes

$$
\frac{z_{3}^{n}}{(\Delta z)^{n-1}}\left(-z_{1} P^{1}+P^{2},-z_{2} P^{1}+P^{3},-z_{3} P^{1}\right),
$$

where $P^{i}=P^{i}\left(1 / z_{3}, z_{1} / z_{3}, z_{2} / z_{3}\right)$.

In a similar way the expression of $p(X)$ in $U_{2}$ is

$$
\frac{z_{3}^{n}}{(\Delta z)^{n-1}}\left(-z_{1} P^{2}+P^{1},-z_{2} P^{2}+P^{3},-z_{3} P^{2}\right)
$$

where $P^{i}=P^{i}\left(z_{1} / z_{3}, 1 / z_{3}, z_{2} / z_{3}\right)$; and in $U_{3}$ is

$$
\frac{z_{3}^{n}}{(\Delta z)^{n-1}}\left(-z_{1} P^{3}+P^{1},-z_{2} P^{3}+P^{2},-z_{3} P^{3}\right)
$$

where $P^{i}=P^{i}\left(z_{1} / z_{3}, z_{2} / z_{3}, 1 / z_{3}\right)$.

In $U_{4}$ we have that $z_{3}^{n+1}\left(P^{1}, P^{2}, P^{3}\right)$ is the expression for $p(X)$ where $P^{i}=P^{i}\left(z_{1}, z_{2}, z_{3}\right)$. The expression for $p(X)$ in the local chart $V_{i}$ is the same as in $U_{i}$ multiplied by $(-1)^{n-1}$.

When we work with the expression of the compactified vector field $p(X)$ in the local charts we shall omit the common factor $1 /(\Delta z)^{n-1}$. We can do that through a rescaling of the time.

We remark that all the points on the sphere at infinity in the coordinates of any local chart have $z_{3}=0$.

In this section we study the behavior of the differential system (1) near the infinity using the Poincaré compactification.
Proof of Proposition 1.1. From (4) the Poincaré compactification of system (1) in the local chart $U_{1}$ is

$$
\begin{aligned}
\dot{z}_{1}= & -z_{2}-7(5 a-4) z_{3}+9(6 a+1) z_{1} z_{3}- \\
& 5(5 a+2) z_{1}^{2} z_{3}, \\
\dot{z}_{2}= & z_{1}+\frac{2}{3}(37 a+11) z_{2} z_{3}-5(5 a+2) z_{2} z_{3} z_{1}, \\
\dot{z}_{3}= & 5(5 a+2)\left(z_{3}^{2}-z_{1} z_{3}^{2}\right) .
\end{aligned}
$$

We look for the equilibria $\left(z_{1}, z_{2}, z_{3}\right)$ with $z_{3}=0$, which are the ones which are at infinity, and we only find the origin, i.e. the endpoint of the positive $x-$ axis, which has eigenvalues 0 and $\pm i$. The system in the local chart $U_{1}$ restricted to infinity $z_{3}=0$ writes $\dot{z}_{1}=-z_{2}, \dot{z}_{2}=z_{1}$. So this equilibrium point at infinity is a center whose period annulus filled of periodic orbits all $U_{1}$. The same occurs at the local chart $V_{1}$.

In short it follows that system (1) has the canonical linear center at the infinity of the local chart $U_{1}$ of the Poincarè ball with the center at the endpoint of the positive $x$-axis.

From (5) the Poincaré compactification of system (1) in the local chart $U_{2}$ is

$$
\begin{aligned}
\dot{z}_{1}= & 5(5 a+2) z_{3}-9(6 a+1) z_{1} z_{3}+z_{1}^{2} z_{2}+ \\
& 7(5 a-4) z_{1}^{2} z_{3}, \\
\dot{z}_{2}= & z_{1}-\frac{1}{3}(88 a+5) z_{2} z_{3}+z_{1} z_{2}^{2}+7(5 a-4) z_{1} z_{2} z_{3}, \\
\dot{z}_{3}= & -(29 a-1) z_{3}^{2}+z_{1} z_{2} z_{3}+7(5 a-4) z_{1} z_{3}^{2} .
\end{aligned}
$$

The equilibria of this system with $z_{3}=0$ are $\left(0, z_{2}, 0\right)$ for all $z_{2} \in \mathbb{R}$. This straight lines filled with equilibria, corresponds to the circle of $\mathbb{S}^{2}$ which is at the end of the plane $x=0$. The same result is obtained working with the local chart $U_{3}$. Note that the local chart $U_{4}$ has no points at infinity. This completes the proof of the proposition.

\section{Invariant algebraic curves}

In this section we prove Proposition 1.2 and Theorem 1.3.

Proof of Proposition 1.2. If $a=-2 / 5$, system (1) becomes

$$
\dot{x}=0, \quad \dot{y}=42 x-x z-\frac{63}{5} y, \quad \dot{z}=x y-\frac{38}{15} z .
$$


Thus $H(x, y, z)=x$ is a first integral, and the flow on each level $H^{-1}(h)$ is determined by the linear differential system

$$
\dot{y}=42 h-h z-\frac{63}{5} y, \quad \dot{z}=h y-\frac{38}{15} z .
$$

This proves statement $(a)$.

For $a=-52 / 149$, we have $\nabla F \cdot(P, Q, R)=$ $k F$, where $F(x, y, z)=149 x^{2}-380 z$ is a Darboux polynomial with cofactor $k=-\frac{380}{149}$. So statement (b) follows.

Let $F(x, y, z)=0$ be an invariant algebraic surface of system (1), and let $m$ be the degree of the polynomial $F$. Then the homogeneous part of degree $m$ of $F$ denoted by $F_{m}$ satisfies

$$
-\frac{\partial F_{m}}{\partial y} x z+\frac{\partial F_{m}}{\partial z} x y=\left(k_{1} x+k_{2} y+k_{3} z\right) F_{m},
$$

where the cofactor of $F=0$ is $k_{0}+k_{1} x+k_{2} y+$ $k_{3} z$. The solution of this linear partial differential equations is either

$$
F_{m}=e^{-\frac{k_{3} y}{x}-\frac{k_{2} z}{x}+k_{1} \tan ^{-1}\left(\frac{y}{z}\right)} h\left(y^{2}+z^{2}\right),
$$

or

$$
F_{m}=e^{-\frac{k_{3} y}{x}+\frac{k_{2} z}{x}-k_{1} \tan ^{-1}\left(\frac{y}{z}\right)} h\left(y^{2}+z^{2}\right),
$$

where $h\left(y^{2}+z^{2}\right)$ is an arbitrary function in the variable $y^{2}+z^{2}$. Since $F_{m}$ must be a homogeneous polynomial, we get that $k_{1}=k_{2}=k_{3}=0$. This proves statement (c).

We take $F$ an arbitrary polynomial of degree at most 6 , i.e.

$$
F=\sum_{i+j=0}^{6} a_{i j} x^{i} y^{j},
$$

and assume that $F=0$ is an invariant algebraic surface with cofactor $k=k_{0} \in \mathbb{R}$, here we have used statement (c). Then the polynomial $\nabla F \cdot(P, Q, R)-$ $k F$ is the zero polynomial, i.e. all the coefficients of this polynomial must be zero. Thus we have a system whose unknowns are the $a_{i j}$ and the $k_{i}$. After tedious but easy computations with the help of an algebraic manipulator such as mathematica or mapple we obtain that the unique solutions of this system are $F=x$ with $a=-2 / 5$ and $F=$ $149 x^{2}-380 z$ with $a=-52 / 149$. This completes the proof of statement $(d)$.
Proof of Theorem 1.3. First we consider the unified chaotic system for $a=-2 / 5$. Then by statement (a) of Proposition 1.2 we know that all the planes $x=h$ with $h \in \mathbb{R}$ are invariant by the flow of the unified chaotic system. This system restricted to the plane $x=h$ becomes

$$
\begin{aligned}
& \dot{y}=\frac{231 h}{2}-\frac{147}{2} y-h z, \\
& \dot{z}=h y-\frac{11}{6} z .
\end{aligned}
$$

The unique equilibrium point of this system is (2). Since its eigenvalues are

$$
\frac{1}{6}\left(-226 \pm \sqrt{46225-36 h^{2}}\right),
$$

it follows easily that such equilibrium point is a stable focus or node if $|h|>215 / 6$ or $|h| \leq 215 / 6$, respectively. Since the differential system (6) on the plane $x=h$ it follows that such focus or node are global, i.e. the filled the whole plane $x=h$. This completes the proof of statement $(a)$.

Now we consider the unified chaotic system for $a=-52 / 149$, and restricting the system on the invariant algebraic surface $z=149 x^{2} / 380$ (see statement (b) of Proposition 1.2) it becomes

$$
\begin{aligned}
& \dot{x}=\frac{190}{149}(y-x), \\
& \dot{y}=\frac{5992}{149} x-\frac{1657}{149} y-\frac{149}{380} x^{3} .
\end{aligned}
$$

It is easy to check that the three equilibria (3) of unified chaotic system for $a=-52 / 149$ are on the invariant algebraic surface. The origin on the surface has the eigenvalues

$$
\frac{1}{298}(-1847 \pm \sqrt{6706009})
$$

so it is a saddle, and the eigenvalue outside the surface is $-380 / 149$. The other two equilibria on the surface have eigenvalues

$$
\frac{1}{298}(-1847 \pm \sqrt{3177791} i)
$$

so they are stable foci, and the eigenvalue outside the surface is again $-380 / 149$. So both foci are local attractors in $\mathbb{R}^{3}$.

The global phase portrait on the invariant algebraic surface of system (7) is topologically equivalent to the one of Figure 2, it has been obtained 
with the program P4 (see Chapters 9 and 10 of [Dumortier et al., 2006]). Moreover, there is numerical evidence that the flow in the interior of the Poincaré ball outside the invariant algebraic surface tends in forward time to the invariant surface. So this proves statement (b).

\section{Hopf bifurcation}

In order to prove that system (1) exhibits a Hopf bifurcation for the unique value $a^{*}$ of its parameter $a$, we will use the following theorem (see [Kuznetsov, 2004], page 178) which shows how to compute the first Lyapunov constant $\ell_{1}\left(p_{1}\right)$ at the equilibrium $p_{1}$.

Lemma 4.1. Assume that the differential system $\dot{\mathbf{x}}=F(\mathbf{x})$ has $p_{0}$ as an equilibrium point. The third order Taylor approximation of the function $F$ around the equilibrium $p_{0}$ is

$$
F(\mathbf{x})=A \mathbf{x}+\frac{1}{2 !} B(\mathbf{x}, \mathbf{x})+\frac{1}{3 !} C(\mathbf{x}, \mathbf{x}, \mathbf{x})+O\left(|\mathbf{x}|^{4}\right) .
$$

Suppose that $A$ has a pair $\pm \lambda i$ of purely imaginary eigenvalues, and that $q$ is the eigenvector of $A$ with eigenvalue $\lambda i$, satisfying $q \bar{q}=1$, where $\bar{q}$ is the conjugate vector of $q$. Let $p$ be the eigenvector of $A^{T}$ with eigenvalue $-\lambda i$ satisfying $\bar{p} q=1$. Let $I$ be the identity matrix. The first Liapunov constant $l_{1}\left(p_{0}\right)$ of the differential system $\dot{\mathbf{x}}=F(\mathbf{x})$ at the equilibrium point $p_{0}$ is

$$
\begin{aligned}
& \frac{1}{2 \lambda} \operatorname{Re}\left(\bar{p} \cdot C(q, q, \bar{q})-2 \bar{p} \cdot B\left(q, A^{-1} B(q, \bar{q})\right)+\right. \\
& \left.\bar{p} \cdot B\left(\bar{q},(2 \lambda i I-A)^{-1} B(q, q)\right)\right) .
\end{aligned}
$$

Proof of Theorem 1.4. The point $p_{-}$is an equilibrium of the unified chaotic system. We translate this equilibrium point to the origin, with the following coordinate change:

$$
\begin{aligned}
& \tilde{x}=x-\sqrt{(9-2 a)(8+a)}, \\
& \tilde{y}=y-\sqrt{(9-2 a)(8+a)}, \\
& \tilde{z}=z+3(9-2 a) .
\end{aligned}
$$

In the new coordinates, again denoted by $(x, y, z)$ instead of $(\tilde{x}, \tilde{y}, \tilde{z})$, system (1) becomes

$$
\begin{aligned}
\dot{x} & =(10+25 a)(y-x)=F_{1}(x, y, z), \\
\dot{y} & =(29 a-1)(y-x)+\sqrt{(9-2 a)(8+a)} z-x z \\
& =F_{2}(x, y, z), \\
\dot{z} & =-\sqrt{(9-2 a)(8+a)}(x+y)+x y-(8+a) z / 3 \\
& =F_{3}(x, y, z) .
\end{aligned}
$$

The linear part of system (9) at the equilibrium point $(0,0,0)$ is given by the matrix $A$ equal to

$$
\left(\begin{array}{ccc}
-5(5 a+2) & 5(5 a+2) & 0 \\
1-29 a & 29 a-1 & K \\
-K & -K & -(a+8) / 3
\end{array}\right)
$$

where $K=\sqrt{(9-2 a)(8+a)}$.

The characteristic polynomial $p(\mu)$ of the ma$\operatorname{trix} A$ is

$$
\begin{aligned}
& -\mu^{3}+\frac{1}{3}(11 a-41) \mu^{2}+\frac{2}{3}\left(5 a^{2}+21 a-152\right) \mu+ \\
& 10\left(10 a^{3}+39 a^{2}-346 a-144\right) .
\end{aligned}
$$

Writing the polynomial

$p(\mu)=(\mu-\rho(a))(\mu-\varepsilon(a)-i \lambda(a))(\mu-\varepsilon(a)+i \lambda(a))$,

we get

$$
\begin{aligned}
\rho(a)= & \frac{\rho_{1}(a)}{9 \sqrt[3]{S}}, \\
\rho_{1}(a)= & -211 a^{2}+(11 \sqrt[3]{S}+524) a-S^{2 / 3}- \\
& 41 \sqrt[3]{S}+1055, \\
\varepsilon(a)= & \frac{1}{18}\left(22 a+\frac{a(211 a-524)-1055}{\sqrt[3]{S}}+\right. \\
\sqrt[3]{S}-82), & -\frac{1}{6 \sqrt{3}}\left|\frac{(524-211 a) a+S^{2 / 3}+1055}{\sqrt[3]{S}}\right|,
\end{aligned}
$$

where

$$
\begin{aligned}
S= & -2 a(a(19633 a+63987)-637044)+ \\
& 9 \sqrt{3} R+425537 \\
R= & \left(( a + 8 ) \left(a \left(a \left(a \left(6306275 a^{2}-8803940 a-\right.\right.\right.\right.\right. \\
& 274063261)+710717860) \\
+ & +546970601)+93753076))^{1 / 2}
\end{aligned}
$$


Then $\rho, \varepsilon$ and $\lambda$ are real if and only if $R \geq 0$. Then it is easy to check that $R \geq 0$ if and only if $a$ belongs to the set

$$
\begin{aligned}
& (-\infty,-8] \cup[-6.9452229210 . ., 4.4844936295 . .] \\
& \cup[4.5065954528 . ., \infty),
\end{aligned}
$$

where the finite endpoints of the previous intervals are zeros of the polynomial $R^{2}$.

In order that a Hopf bifurcation can take place at the origin of the differential system (9) $\varepsilon$ must be zero, but an easy study shows that this only occurs when

$$
a=a^{*}=-0.0136810441173477 \ldots
$$

Then the eigenvalues of the matrix $A$ are

$$
\begin{aligned}
\rho\left(a^{*}\right) & =-13.7168304950 . ., \\
\pm \lambda\left(a^{*}\right) i & = \pm 10.0759239798 . . i .
\end{aligned}
$$

Now the Hopf bifurcation will take place at the origin $O$ of the differential system (9) when $a=a^{*}$ if the Lyapunov constant $\ell_{1}(O)$ is not zero. We compute $\ell_{1}(O)$ using the Lemma 4.1. We need to write the function

$$
\begin{aligned}
F(\mathbf{x}) & =F(x, y, z) \\
& =\left(F_{1}(x, y, z), F_{2}(x, y, z), F_{3}(x, y, z)\right)
\end{aligned}
$$

of the differential system (9) as it appears in (8). We already now the matrix $A$, then an easy computation shows that the bilinear form $B(\mathbf{x}, \mathbf{y})$ of $(8)$ for our system (9) is

$$
B((x, y, z),(u, v, w))=(0,-w x-u z, v x+u y) .
$$

Since the polynomial differential system (9) is quadratic it follows that the trilinear form $C(\mathbf{x}, \mathbf{y}, \mathbf{z})$ of (8) is zero.

Computing the normalized eigenvector $q$ of $A$, associated to the eigenvalue $\lambda i=10.0759239798 . . i$, we obtain

$$
q=\left(\begin{array}{c}
-0.2649114919 . .-0.2906456499 . . i \\
0.0383118865 . .-0.5670212213 . . i \\
0.7227490169 . .
\end{array}\right)
$$

The normalized adjoint eigenvector of the transpose matrix $A$ with the eigenvalue $-\lambda i$ is

$$
p=\left(\begin{array}{c}
-0.2100011995 . .-0.26682108409 . . i \\
0.1076431231 . .-0.69924528074 . . i \\
0.5732025206 . .-0.0607327918 . . i
\end{array}\right) \text {. }
$$

From Lemma 4.1 in order to compute $l_{1}(O)$ we must compute $-2 \bar{p} \cdot B\left(q, A^{-1} B(q, \bar{q})\right)$ and $\bar{p}$. $\left.B\left(\bar{q},(2 \lambda i I-A)^{-1} B(q, q)\right)\right)$. We have

$$
\begin{aligned}
& -2 \bar{p} \cdot B\left(q, A^{-1} B(q, \bar{q})\right)= \\
& 0.0078908121 . .-0.0706414304 . . i,
\end{aligned}
$$

and

$$
\begin{aligned}
& \left.\bar{p} \cdot B\left(\bar{q},(2 \lambda i I-A)^{-1} B(q, q)\right)\right)= \\
& -0.00335845379 . .+0.0253657537 . . i .
\end{aligned}
$$

Then

$$
\begin{aligned}
l_{1}(O)= & \frac{1}{2 \lambda} \operatorname{Re}\left(-2 \bar{p} \cdot B\left(q, A^{-1} B(q, \bar{q})\right)+\right. \\
& \left.\bar{p} \cdot B\left(\bar{q},(2 \lambda i I-A)^{-1} B(q, q)\right)\right) \\
= & 0.0002249103 . .
\end{aligned}
$$

which is different from zero. This finishes the proof of Theorem 1.4.

\section{Acknowledgments}

The first author is partially supported by a MINECO/FEDER grant MTM2008-03437 and MTM2013-40998-P, an AGAUR grant number 2014SGR-568, an ICREA Academia, the grants FP7-PEOPLE-2012-IRSES 318999 and 316338, and UNAB 13-4E-1604.

\section{References}

Algaba, A. Fernandez-Sanchez, F., Merino, M. \& Rodriguez-Luis, A.J. [2013] "Chen's attractor exists if Lorenz repulsor exists: The Chen system is a special case of the Lorenz system," Chaos 23, 033108..

Buzzi, C.A., Llibre, J. \& da Silva, P.R. [2007] "3dimensional Hopf bifurcation via averaging theory," Discrete, Continuous Dynamical Systems, Series A 17, 529-540..

Celikovsky, S. \& Vaecek, A. [403-424] "Bilinear systems and chaos," Kybernetika 30,..

Celikovsky, S. \& Chen, G. [1789-1812] "On a generalized Lorenz canonical form of chaotic systems," Int. J. Bifurcation and Chaos 12,..

Celikovsky, S. \& Chen, G. [1271- 1276] "On the generalized Lorenz canonical form," Chaos Solit. Fract. 26, .. 
Chen, G. [2013] "The Chen system revisited," $D y$ namics of Continuous, Discrete and Impulsive Systems Series B: Applications \& Algorithms 20, 691-696.

Chen, G. \& Ueta, T. [1999] "Yet another chaotic attractor," Internat. J. Bifur. Chaos Appl. Sci. Engrg. 9, 1465-1466.

Cima, A. \& Llibre, J. [1990] "Bounded polynomial vector fields," Trans. Amer. Math. Soc. 318, $557-579$.

Dumortier, F., Llibre, J. \& Artés, J.C., [2006] Qualitative theory of planar differential systems UniversiText 5, Springer-Verlag, New York.

Kuznetsov, Y., [2004] Elements of applied bifurcation theory Applied Mathematical Sciences 112, Springer-Verlag, New York.

Leonov, G.A. \& Kuznetsov, N.V. [2015] "On differences and similarities in the analysis of Lorenz, Chen, and Lu systems," Appl. Math. Comput. 256, 334-343.

Li, C. \& Chen, G. [2003] "A note on Hopf bifurcation in Chen's system," Internat. J. Bifur. Chaos Appl. Sci. Engrg. 13, 1609-1615.

Lin, Z., Yu, S., Lü, J., Cai, S. \& Chen, G. [2015] "Design and ARM-Embedded Implementation of A Chaotic Map-Based RealTime Secure Video Communication System," IEEE Trans. Circuits Syst. Video Technol. DOI: 10.1109/TCSVT.2014.2369711, 14 pp.

Llibre, J., Messias, M. \& Silva, P. [2010] "Global dynamics of the Lorenz systems with invariant algebraic surfaces," Int. J. Bifurcation and Chaos 20, 3137-3155.

Llibre, J., Messias, M. \& Silva, P. [2012] "Global dynamics in the Poincarè ball of the Chen system having invariant algebraic surfaces," Internat. J. Bifur. Chaos Appl. Sci. Engrg. 22, 1250154-17 pp.

Lorenz,E.N. [1963] "Deterministic non-periodic flow," J. Atmos. Sci. 20, 130-141.

Lu, J., Chen, G. [2006] "Generating multiscroll Chaotic attractors: Theories, Methods and Applications," Internat. J. Bifur. Chaos Appl. Sci. Engrg. 16, 775-858.
Lu, J., Chen, G., Cheng, D. \& Celikovsky, S. [2002] "Bridge the gap between the Lorenz system and the Chen system," Internat. J. Bifur. Chaos Appl. Sci. Engrg. 12, 2917-2926.

Roschin, N. [1978] "Dangerous stability boundaries in the Lorenz model," Prikl. Mat. Mekh. 42, 950-952 (in Russian).

Tucker, W. [2002] "A Rigorous ODE Solver and Smale's 14th Problem," Foundations of Computational Mathematics 2, 53-117. 